\title{
Adaptación al Español de la Escala de Desempeño Cívico de Coleman y Borman (2000) y Análisis de la Estructura Empírica del Constructo
}

\section{Spanish Adaptation of the Citizenship Performance Questionnaire by Coleman \& Borman (2000) and an Analysis of the Empiric Structure of the Construct}

\author{
Luis Díaz-Vilela, Dolores Díaz-Cabrera, Rosa Isla-Díaz, Estefanía Hernández-Fernaud \\ y Christian Rosales-Sánchez \\ Universidad de La Laguna
}

\begin{abstract}
Resumen. Este artículo presenta una adaptación del cuestionario de desempeño cívico de Coleman y Borman (2000) y un análisis de su estructura empírica. Una vez traducido y adaptado, el cuestionario se aplicó a una muestra de 135 empleados públicos de una unidad completa del Gobierno Autonómico de Canarias. Los resultados indican que el cuestionario tiene una alta consistencia interna, aunque parece medir tres dimensiones relacionadas entre sí. Dos de estas dimensiones son altamente estables y coinciden con las propuestas por LePine, Erez y Johnson (2002), Cumplimiento Generalizado y Altruismo, mientras que la tercera es de difícil interpretación. Un análisis factorial de segundo orden indica que existe una única dimensión subyacente de desempeño cívico. Estos resultados tienen implicaciones teóricas y prácticas que ayudan a la interpretación y diagnóstico del dominio.

Palabras clave: desempeño cívico, desempeño contextual, conductas de ciudadanía organizacional, escala de desempeño cívico.
\end{abstract}

\begin{abstract}
This article presents a Spanish adaption of the citizenship performance questionnaire of Coleman and Borman (2000) an analysis of its empirical structure. Once the original questionnaire was translated and adapted to a Spanish public administration context, it was administered to a sample of 135 public employees representing the complete staff ofa ' Autonomic Government unit. Results show a high internal consistency of the questionnaire, although it seems to measure three related dimensions. Two of these dimensions are highly stable and coincide with those proposed by LePine, Erez, and Johnson (2002), Generalized Compliance and Altruism, while the third dimension is difficult to interpret. A second order factor analysis supports the existence of a unique underlying dimension of citizenship performance. These results have theoretical as well as practical implications that may help the interpretation and diagnosis in this domain.

Keywords: citizenship performance, contextual performance, organizational citizenship behaviour, citizenship performance scale.
\end{abstract}

El desempeño laboral ha sido uno de los dominios más investigados y en el que se han obtenido avances muy relevantes en los últimos años en el área de la Psicología del Trabajo y de las Organizaciones. Entre estos avances se encuentra el reconocimiento de la importancia que tiene la inclusión en una evaluación del desempeño de todas aquellas conductas que repercuten, positiva o negativamente, en los resultados organizacionales, y no limitarse a las tareas asignadas al puesto y posición específica (Salgado y Cabal, 2011). Los modelos de desempeño agrupan estas conductas en tres grandes dimensiones: desempeño de

La correspondencia sobre este artículo se enviará a Luis Díaz Vilela, Facultad de Psicología, Campus de Guajara, 38205, La Laguna, Tenerife.E-mail: ldiazv@ull.es. tareas, desempeño cívico y conductas contraproductivas (Ployhart, Schneider y Schmitt, 2006; Salgado y Cabal, 2011; Viswesvaran y Ones, 2000). Una evaluación del desempeño debería incluir, por tanto, medidas de desempeño de estos tres ámbitos.

El desempeño de tarea se define como la efectividad con la que un empleado ejecuta las actividades que contribuyen al núcleo técnico de la organización (Borman y Motowidlo, 1997; Viswesvaran y Ones, 2000) o la competencia con la que los empleados desempeñan las actividades reconocidas formalmente como constituyentes de sus puestos de trabajo (Motowidlo y Schmit, 1999).

Las conductas contraproductivas son aquellas conductas destructivas voluntarias que violan normas importantes de la organización y que amenazan el 
bienestar de ésta, de sus miembros, o de ambos (Ployhart et al., 2006; Salgado y Cabal, 2011). Se puede decir que se trata de un conjunto de conductas que generan "contra - desempeño".

El desempeño cívico constituye el objeto de este artículo, y más específicamente la adaptación de la escala utilizada por Coleman y Borman para investigar la estructura del dominio (Coleman y Borman, 2000). El desempeño cívico se define como la realización de un conjunto de conductas discrecionales que, generalmente, no forman parte del rol formal y que no son reconocidas directa o explícitamente en los sistemas de compensación. En conjunto, estas conductas promueven el funcionamiento eficaz de la organización (Bateman y Organ, 1983; Borman y Motowidlo, 1997; Organ, 1988; Organ y Ryan, 1995; Ployhart et al., 2006). En otras palabras, son conductas que contribuyen al mantenimiento del sistema social facilitando el logro de las metas organizacionales. Son conductas dirigidas a crear un ambiente organizacional de apoyo social y psicológico, en el que el núcleo técnico debe funcionar (Borman y Motowidlo, 1993; Witman, Van Rooy y Viswesvaran, 2010). Originalmente, Bateman y Organ (1983) definieron la "Ciudadanía" como cualquiera de esos gestos que lubrican la maquinaria social de la organización pero que no se incluyen directamente en la noción de desempeño de tarea. Concretamente, se refiere a conductas tales como la voluntariedad para realizar tareas que no se incluyen en la definición formal (explícita) del trabajo, la ayuda y la cooperación con los compañeros, el cumplimiento de las normas y procedimientos establecidos o la aportación de sugerencias sobre cómo mejorar los procedimientos de trabajo.

El desempeño cívico se diferencia del desempeño de tarea en tres aspectos importantes (Borman, Penner, Allen y Motowidlo, 2001; Dorsey, Cortina y Luchman, 2010; Organ y Ryan, 1995; Ployhart et al., 2006). Primero, las conductas relacionadas con las tareas varían entre los puestos de trabajo, mientras que las conductas de ciudadanía organizacional son más similares entre puestos. Segundo, las tareas tienden a estar más explícita y formalmente establecidas en los requerimientos del puesto que las actividades cívicas. Finalmente, los antecedentes del desempeño de tarea parecen implicar en mayor medida habilidades y destrezas, mientras que los antecedentes del desempeño contextual son, en mayor grado, rasgos de personalidad.

El desempeño cívico depende de actividades tales como ayudar a los compañeros en sus tareas laborales, apoyar a la organización o responsabilizarse de tareas adicionales al puesto de trabajo de forma voluntaria (Borman et al., 2001). Esta variedad de conductas referidas a diferentes ámbitos del contexto laboral fueron categorizadas por Borman y Motowidlo (1993, 1997) en su conocida taxonomía de cinco dimensiones: "Persistir con entusiasmo y esfuerzo extra para com- pletar con éxito las tareas propias"; "Tener la voluntad para hacer tareas que no corresponden formalmente al puesto" "Ayudar a otros y cooperar con ellos"; "Seguir las reglas y procedimientos organizacionales" $\mathrm{y}$ "Favorecer, apoyar y defender los objetivos organizacionales". A partir de esta taxonomía, Coleman y Borman (2000) analizaron las respuestas de 44 miembros de la Society for Industrial and Organizational Psychology (SIOP), que ordenaron 27 conductas de desempeño cívico. Seguidamente, usando los resultados empíricos de análisis factorial, escalamiento multidimensional y análisis de clusters, los autores propusieron un modelo racional integrado, que contempla tres grandes dimensiones: desempeño cívico interpersonal, desempeño cívico organizacional, y desempeño cívico relacionado con el puesto/tarea.

En un trabajo anterior, siguiendo un diseño experimental, Werner (1994) encontró que los supervisores experimentales evaluaron el desempeño de trabajadores ficticios según tres dimensiones: desempeño en la tarea; desempeño cívico orientado a beneficiar a la organización $(O C B O)$ y desempeño cívico dirigido a beneficiar a individuos $(O C B I)$. Estas dos últimas dimensiones se correspondían con las de Cumplimiento Generalizado y Altruismo encontradas anteriormente por Smith, Organ y Near (1983), a partir de un estudio empírico basado en el análisis de componentes principales de la matriz de correlaciones de una escala de 16 elementos. El componente de Altruismo aglutinó conductas relacionadas con ayudar a compañeros, mientras que el componente de Cumplimiento Generalizado saturó principalmente las preguntas relacionadas con el cumplimiento de horarios y pautas de trabajo. En la misma línea, Hogan, Rybicki, Motowidlo y Borman (1998), con un cuestionario de 55 preguntas, aplicado a 214 casos, encontraron una estructura de dos factores: Dedicación al trabajo y Facilitación interpersonal. Sin embargo, en el segundo estudio de la misma investigación, Hogan et al. (1998), usaron un cuestionario diferente en el que midieron las cinco dimensiones de Borman y Motowidlo (1993; 1997), a través de cuatro preguntas por dimensión. En su estudio, Hogan y colaboradores no comprobaron la estructura empírica de su cuestionario, dando por válida la clasificación original.

Otros autores han llevado a cabo investigaciones sobre este dominio bajo la hipótesis de su unidimensionalidad. Por ejemplo Poropat y Jones (2009), a partir de la clasificación tridimensional aportada por Coleman y Borman (2000), desarrollaron un cuestionario que contenía cuatro elementos de cada dimensión. Este cuestionario se usó para evaluar el desempeño de 298 estudiantes de grado tras cuatro semanas de trabajo en un proyecto. Los evaluadores en este trabajo fueron los propios compañeros de clase/proyecto de los evaluados. Según estos autores, sus resultados apoyarían "ostensiblemente" la hipótesis defendida por Hoffman, Blair, Meriac y Woehr (2007), pues su cues- 
tionario de Desempeño Cívico mediría un único factor latente. En realidad, Hoffman et al. (2007) se refieren a la dicotomía entre la existencia de un único conjunto de conductas de ciudadanía organizacional, o de varias dimensiones que son en realidad indicadores imperfectos de un constructo simple o único. Si tenemos esto en cuenta, el resultado unidimensional del análisis factorial confirmatorio obtenido por Poropat y Jones (2009) apoyaría, más bien, la hipótesis de la agregación: existe un único conjunto de conductas y, por tanto, se pueden promediar todas las puntuaciones de un cuestionario para obtener una única puntuación de desempeño contextual. Sin embargo, esta afirmación es incompatible con los resultados de fiabilidad que estos mismos autores informan, con valores iguales o inferiores a $\alpha$ $=0.90$.

Los ejemplos presentados ponen de manifiesto algunos de los problemas que encontramos en la literatura relacionada con el desempeño cívico. Por una parte, no existe una nomenclatura clara y rigurosa del dominio, apareciendo a veces como conductas discrecionales, otras como conductas de ciudadanía organizacional, otras como desempeño contextual y otras como desempeño cívico. Por otra parte, no existe un acuerdo sobre la estructura interna del constructo, ni siquiera sobre su uni/pluri-dimensionalidad. Finalmente, no existe un conjunto de preguntas, más o menos estandarizadas, susceptibles de incorporar en un cuestionario de desempeño cívico, sino más bien, proliferan las escalas diferentes. LePine, Erez y Johnson (2002) encontraron 40 medidas diferentes de conductas de ciudanía organizacional cuya validez no fue suficientemente analizada y rara vez se ha informado de los contenidos de los cuestionarios.

En la última década, se ha realizado un importante esfuerzo para clarificar y conceptuar las dimensiones componentes del desempeño cívico. LePine et al. (2002) proponen que el desempeño cívico debe ser conceptuado como un constructo latente. A partir de su meta-análisis concluyen que las cinco dimensiones de conductas cívicas organizacionales son, en realidad, equivalentes. El constructo resultante, Conductas de Ciudadanía Organizacional (OCB), debería, según estos autores, redefinirse como "una tendencia general a cooperar y ayudar en los entornos organizacionales" (p. 61). Esta definición incluye claramente las dos dimensiones encontradas por Smith et al. (1983), Werner (1994) y Hogan et al. (1998) en los trabajos citados anteriormente. Sin embargo, la asunción de la existencia de un constructo latente implicaría que las correlaciones entre ambas dimensiones deben ser considerablemente altas, como de hecho ocurre, y en todo caso, un análisis factorial de segundo orden debería resultar en un único componente. LePine et al. (2002) reconocen que la evidencia empírica disponible es bastante limitada y que, por tanto, no puede usarse para apoyar el modelo de constructo latente frente a otro modelo alternativo, como el del constructo agregado.
No obstante, para medir adecuadamente este constructo agregado sería necesario desarrollar preguntas relacionadas con actividades que contribuyan a los fines de la organización concreta bajo evaluación. La puntuación en este constructo sería, entonces, la agregación de las puntuaciones en estas actividades (LePine et al., 2002).

Años después, Hoffman et al. (2007) ampliaron la revisión meta-analítica de la literatura existente sobre OCB usando análisis factorial confirmatorio. El objetivo de su estudio fue investigar la dimensionalidad del desempeño cívico y examinar su relación, como constructo latente, con el desempeño de la tarea, así como con variables actitudinales (satisfacción, compromiso, justicia distributiva, de interacción y de procedimiento). Los resultados mostraron un modelo de un factor único de OCB y un factor de desempeño en la tarea, fuertemente relacionados. Así, estos autores señalan que el desempeño cívico se caracteriza mejor por un único factor de orden superior. Más concretamente, indican que las subdimensiones del OCB son indicadores de un constructo más general.

Aunque obviásemos la condición propuesta por LePine et al. (2002) en relación a la metodología de investigación del constructo agregado, los procedimientos estadísticos más importantes para su comprobación serían el análisis de fiabilidad (consistencia interna) y el análisis factorial. En la mayoría de los casos estudiados, el coeficiente de fiabilidad obtenido ha sido el $\alpha$ de Cronbach. Sin embargo, además de no informar sobre la aditividad de los elementos que componen las escalas usadas, no se ha tenido en cuenta que este índice es un mal indicador de la estructura de los datos. En este sentido, Revelle y Zinbarg (2009) proponen la utilización de las pruebas Omega total $-\omega_{\mathrm{t}}$ (McDonald, 1978) y Omega jerárquica $-\omega_{\mathrm{h}}$ (McDonald, 1999). Omega total da información sobre la proporción de la varianza del test que se debe a todos los factores comunes a los elementos que lo componen. Sólo cuando $\omega_{\mathrm{t}}$ es igual a $\alpha$, el test puede considerarse unidimensional y, por tanto, la hipótesis del constructo agregado tendría apoyo. Omega jerárquica, por su parte, da información sobre la proporción de varianza del test debida a un único factor general. Esta información es importante y relevante aquí porque se refiere a la precisión con que las puntuaciones totales del test son estimaciones de una variable latente común a todos los elementos del mismo. Si este índice fuese alto, la hipótesis del constructo agregado tendría apoyo empírico.

Por otra parte, la hipótesis de un constructo agregado tendría más apoyo si las soluciones factoriales con un único componente tuvieran mejor ajuste que aquellas con varios factores. Además, si las soluciones factoriales con varios factores generaran factores intercorrelacionados, la hipótesis del factor latente cobraría fuerza. Desafortunadamente, salvo en los casos en los que se aplican modelos de medida y ecuaciones estruc- 
turales, rara vez se informa sobre la bondad de ajuste de los resultados factoriales. Además, cuando se informa sobre análisis factoriales, normalmente lo que se ha utilizado han sido análisis de componentes principales $\mathrm{y}$, la mayor parte de las veces, con rotaciones ortogonales.

En este trabajo pretendemos ofrecer un cuestionario que represente con la mayor fidelidad posible el listado de ítems utilizado por Coleman y Borman (2000), pero aplicado, en esta ocasión, a trabajadores en un entorno concreto y en una situación real de evaluación del desempeño. Además, como segundo objetivo, pretendemos ofrecer información sobre la estructura del constructo desempeño cívico basándonos en herramientas estadísticas adecuadas a los datos.

Este cuestionario puede ser una herramienta útil para los profesionales de los Recursos Humanos que pretendan no sólo evaluar el desempeño, sino también diagnosticar las necesidades de formación del personal en el ámbito del desempeño laboral. Especialmente por haber sido adaptado al español, lengua en la que hay una escasez de instrumentos de evaluación del desempeño cívico contrastados empíricamente.

\section{Método}

\section{Participantes}

El estudio se realizó con una muestra de 135 empleados públicos (29.6\% hombres y $70.4 \%$ mujeres) de una unidad de la Administración Autonómica de las Islas Canarias, cuya función principal es la de desarrollar acciones de estudio, propuesta, impulso y ejecución de la política del Gobierno Autonómico en las materias de ordenación, promoción e infraestructura turísticas, así como de costas. Estos 135 empleados suponen el total de la plantilla de la Unidad, que se estructura en 18 unidades menores (servicios, direcciones generales y unidades de apoyo) y 25 puestos de trabajo diferentes, que se agruparon en ocho familias de puestos: Administrativos (37\%); Aparejadores (2.2\%); Inspectores (3.7\%); Jefes Sección/Servicio (22.2\%); Operadores Informáticos (3.7\%); Secretarias (4.4\%); Subalternos $(8.9 \%)$ y Titulados $(17.8 \%)$.

\section{Instrumento}

El cuestionario de desempeño cívico utilizado consistió en una adaptación al español del que fue desarrollado por Coleman y Borman (2000). Esta adaptación fue llevada a cabo en varias fases. En primer lugar, se realizó una primera traducción del cuestionario original, siguiendo el criterio de mantener exactamente el sentido de cada pregunta, variando sólo los giros idiomáticos. En esta fase participaron seis investigadores expertos que discutieron la adaptación de cada uno de los elementos. A continuación, un comité de expertos de la Administración evaluó la claridad de las preguntas y propuso, en su caso, alternativas de mejora.

El cuestionario utilizado finalmente estuvo compuesto por 27 preguntas con escalas gráficas asociadas de 6 puntos y dos anclajes extremos. Se pidió a los participantes que contestaran cada pregunta en función de lo que creían que describía mejor su forma de comportarse en el trabajo.

\section{Procedimiento}

Cada uno de los empleados recibió el cuestionario de auto evaluación del desempeño cívico junto con otros dos cuestionarios que no se usarán en este artículo: uno de auto evaluación del desempeño en la tarea, específico para cada grupo profesional y un cuestionario sobre la validez percibida del cuestionario de desempeño en la tarea y la sinceridad en la respuesta.

Se pidió a cada participante que contestase sus cuestionarios y los devolviese al equipo de investigación. Cada cuestionario fue identificado y el equipo de investigación garantizó la confidencialidad de los datos individuales. Tras un plazo de 15 días se recogieron los cuestionarios cumplimentados y se procedió al análisis de tendencias de respuestas. Los criterios utilizados para detectar casos con tendencias anormales de respuesta fueron: media global por caso alta (una desviación típica por encima de la media de la muestra); la suma de productos de la alternativa elegida por la frecuencia de elecciones alta (una desviación típica por encima de la media de la muestra); número muy reducido de alternativas elegidas (una desviación típica por debajo de la media de la muestra). Aquellos que cumplían con más de uno de estos criterios fueron informados de la existencia de problemas con sus datos y fueron visitados por un técnico del equipo de investigación. Cada caso obtuvo una explicación de las razones por las que habían sido rechazados sus datos y pudo corregir o mantener sus respuestas originales. Los datos sobre los que se trabajará en este estudio han sido confirmados por los participantes, aunque todavía exista un $16.3 \%$ de casos que cumple más de uno de estos criterios.

\section{Análisis de datos}

Los dos objetivos de este trabajo fueron abordados con dos metodologías de análisis de datos. En primer lugar, pretendemos analizar la adecuación psicométrica del cuestionario desarrollado. Dado que el cálculo de la consistencia interna por el método Cronbach no es un buen indicador de la estructura de los datos (Revelle y Zinbarg, 2009), aplicamos el coeficiente Omega, basado en el análisis factorial de la escala y el de Guttman, que analiza la estabilidad de dos mitades 
de la escala. Los resultados de Omega jerárquica $\left(\omega_{\mathrm{h}}\right)$ y Omega total $\left(\omega_{\mathrm{t}}\right)$ son relevantes para este trabajo. La primera, $\omega_{\mathrm{h}}$, es la proporción de varianza del cuestionario debida a un factor general, lo que nos informa sobre la precisión con la que las puntuaciones totales de la escala son una estimación de una variable latente común a todos los elementos de la misma. La segunda, $\omega_{\mathrm{t}}$, es la proporción de varianza del cuestionario debida a todos los factores comunes y tiene el mismo valor que $\alpha$ cuando el cuestionario es unidimensional. Entre los resultados de la prueba de Guttman, $\lambda_{6}$ es el límite que hemos elegido por ser también un indicador de la existencia de un factor común a todos los elementos, pues considera la cantidad de varianza de cada elemento que puede explicarse por la regresión lineal de todos los demás elementos.

El segundo objetivo de este artículo consiste en el análisis de la estructura del constructo medido por el cuestionario. Para cumplir con este objetivo se desarrolló un procedimiento de análisis factoriales aplicados sobre la matriz de correlaciones policóricas y un análisis factorial de segundo orden sobre las puntuaciones factoriales obtenidas. Las correlaciones policóricas son adecuadas cuando asumimos que las respuestas ordinales de los participantes son reflejo de elecciones que éstos hacen a partir de una concepción continua del constructo medido. Esto es, son útiles para estimar la correlación entre variables latentes continuas cuya distribución teórica es normal, observadas a partir de variables ordinales. Para este tipo de escalas es más recomendable estimar la matriz de correlaciones policóricas (Choi, Peters y Mueller, 2010; Ekström, 2008, 2009; Juras y Pasaric, 2006; Uebersax, 2006) y aplicar el análisis factorial sobre esta matriz. La escala de las variables medidas en el cuestionario es ordinal, pues las respuestas son valores enteros entre 1 y 6 puntos, por lo que los análisis de componentes principales y factoriales se aplicarán sobre $\mathbf{R}_{\text {(policórica) }}$. Finalmente, dentro de este segundo objetivo, se llevó a cabo un análisis factorial de segundo orden sobre las puntuaciones factoriales obtenidas en los análisis previos con el fin de comprobar si la estructura de un factor latente es adecuada.

\section{Resultados}

La Tabla 1 muestra los principales estadísticos sobre la distribución de los elementos y la fiabilidad de la escala. Aunque las medias de los elementos son muy similares entre sí, al aplicar la $\mathrm{T}^{2}$ de Hotelling obtenemos valores significativos, por lo que asumiremos que estas medias no son iguales. En general, las respuestas están muy sesgadas a la derecha, con una media mínima, por elemento, de 4.22 y máxima de 5.15 , indicando una tendencia de respuesta hacia los valores máximos ("Es lo que le caracteriza"). Todas las pruebas de contraste de normalidad aplicadas a las distribuciones de cada elemento dieron resultados similares: ninguna de las distribuciones de respuesta de los elementos del cuestionario puede ser considerada normal. Se informa sólo sobre las puntuaciones $Z$ de la prueba de Kolmogorov - Smirnov, pues son más fácilmente interpretables.

Finalmente, cabe destacar que la distribución de las medias del cuestionario (puntuación media agregada de elementos en el cuestionario) no se separa de la normalidad ( $Z=0.793$; n.s.).

La fiabilidad del cuestionario es relativamente alta si atendemos al coeficiente de Cronbach $(\alpha=0.96)$. Todos los elementos se comportan adecuadamente, de manera que ninguna exclusión mejora significativamente la fiabilidad de la escala. El elemento con peor comportamiento es el 11 ("No quejarse de las condiciones de trabajo en la Consejería."), cuya redacción puede haber causado problemas de comprensión en algunos casos (construcción negativa con valor positivo). Además, las pruebas de aditividad resultaron adecuadas, con una F para la interacción sujeto $\times$ elemento no significativa, y una potencia de elevación de las observaciones de aproximadamente 1.5.

Los resultados de los coeficientes Omega y de Guttman se presentan en el pie de la Tabla 1. En primer lugar apreciamos que $\lambda_{6}$ de Guttman tiene un valor ligeramente superior al $\alpha$ de Cronbach, lo que podría indicar que la escala se explica mejor con un único factor subyacente. Este resultado es congruente con el valor de Omega jerárquica, $\omega_{h}$, que indica que un único factor da cuenta del $79 \%$ de la varianza de los 27 elementos. Además, el valor de Omega total, $\omega_{t}$, es igual al de $\alpha$ de Cronbach, apoyando también la unidimensionalidad del cuestionario. Sin embargo, el análisis de fiabilidad desarrollado en el software estadístico R ( $R$ Development Core Team, 2008), que realiza un análisis factorial con la mejor solución alternativa a la unidimensionalidad, sugiere una solución consistente en tres factores. En la Tabla 2 se presentan los estadísticos de ambos resultados, soluciones unidimensional y tridimensional. Como puede verse, todos los indicadores mejoran con la solución de tres factores, aunque esta mejoría no es sustancial.

El análisis factorial aplicado por el procedimiento Omega de análisis de la fiabilidad, se apoya en la matriz $\mathbf{R}$ de correlaciones de Pearson. Sin embargo, esta matriz es adecuada para variables de nivel de intervalo, mientras que la escala de las variables de este trabajo es ordinal. En consecuencia, aplicamos un Análisis Paralelo sobre la matriz $\mathbf{R}_{\text {(policórica) }}$ para estimar el número de factores y componentes contenidos en el cuestionario. El resultado de este análisis es congruente con los resultados del análisis de fiabilidad. Parece existir un componente principal y tres factores. Según estos resultados procedimos a aplicar un análisis de Componentes Principales extrayendo un único componente, y un Análisis Factorial extrayendo tres factores (método de mínimos cuadrados ponderados), rotados 
Tabla 1. Estadísticos sobre la distribución de los elementos y la fiabilidad de la escala

\begin{tabular}{|c|c|c|c|c|c|c|c|c|c|c|c|}
\hline \multirow{2}{*}{\multicolumn{2}{|c|}{ Elemento de OCB }} & \multicolumn{5}{|c|}{ Estadísticos descriptivos } & \multirow[b]{2}{*}{$\begin{array}{l}\text { Asimetría } \\
\text { (ET=.209) }\end{array}$} & \multirow[b]{2}{*}{$\begin{array}{l}\text { Curtosis } \\
\text { (ET=.414) }\end{array}$} & \multirow{2}{*}{$\begin{array}{c}\mathrm{K}-\mathrm{S} \\
\mathrm{Z}\end{array}$} & \multicolumn{2}{|c|}{ Fiabilidad } \\
\hline & & $\mathrm{N}$ & Mín. & Máx. & Media & D. T. & & & & $\begin{array}{c}\mathrm{R}^{2} \\
\text { Múlt. }\end{array}$ & $\begin{array}{l}\alpha \text { sin el } \\
\text { elemento }\end{array}$ \\
\hline 01 & Manifestar un entusiasmo constante al realizar su trabajo. & 135 & 1 & 6 & 4.54 & 1.091 & -.438 & -.0392 & $2.077 * * *$ & 0.69 & 0.95 \\
\hline 02 & Mostrar un esfuerzo superior al normal en su trabajo. & 135 & 1 & 6 & 4.41 & 1.122 & -.363 & -.3232 & $2.183 * * *$ & 0.58 & 0.95 \\
\hline 03 & Mostrar disposición para llevar a cabo tareas que no forman parte de su puesto de trabajo. & 135 & 1 & 6 & 4.59 & 1.061 & -.589 & .1402 & $2.671 * * *$ & 0.56 & 0.95 \\
\hline 04 & Ayudar a otros compañeros en la Consejería. & 135 & 1 & 6 & 5.03 & .889 & -.964 & 1.9822 & $2.729 * * *$ & 0.75 & 0.95 \\
\hline 05 & Cooperar con otros compañeros de la Consejería. & 135 & 1 & 6 & 5.03 & .954 & -1.055 & 1.494 & $2.911 * * *$ & 0.82 & 0.95 \\
\hline 06 & Seguir las normas y procedimientos de la Administración. & 135 & 2 & 6 & 5.15 & .833 & -.914 & .899 & $2.838 * * *$ & 0.60 & 0.95 \\
\hline 07 & Asumir, apoyar o defender los objetivos de la Consejería. & 134 & 2 & 6 & 4.57 & 1.106 & -.445 & -.466 & $2.338 * * *$ & 0.72 & 0.95 \\
\hline 08 & Ayudar desinteresadamente a otros compañeros de la Consejería. & 135 & 2 & 6 & 5.11 & .903 & -.716 & -.093 & $2.931 * * *$ & 0.74 & 0.95 \\
\hline 09 & Esmerarse en favorecer a la Administración. & 135 & 2 & 6 & 4.61 & 1.022 & -.273 & -.686 & $2.352 * * *$ & 0.71 & 0.95 \\
\hline 10 & Mantener una actitud positiva hacia la Administración. & 135 & 2 & 6 & 4.68 & .975 & -.401 & -.373 & $2.650 * * *$ & 0.78 & 0.95 \\
\hline 11 & No quejarse de las condiciones de trabajo en la Consejería. & 135 & 1 & 6 & 4.23 & 1.051 & -.435 & .244 & $2.214 * * *$ & 0.32 & 0.96 \\
\hline 12 & Compartir información con los demás sobre futuros eventos, actividades, acciones, etc. & 135 & 1 & 6 & 4.58 & 1.096 & -.857 & .876 & $2.732 * * *$ & 0.49 & 0.95 \\
\hline 13 & Participar responsablemente en la Consejería. & 135 & 2 & 6 & 4.85 & .950 & -.545 & -.319 & $2.828 * * *$ & 0.57 & 0.95 \\
\hline 14 & Demostrar lealtad a la Administración. & 135 & 2 & 6 & 4.95 & .972 & -.538 & -.522 & $2.509 * * *$ & 0.60 & 0.95 \\
\hline 15 & Promover, promocionar y defender la Consejería. & 134 & 1 & 6 & 4.28 & 1.218 & -.333 & -.417 & $1.877 * * *$ & 0.73 & 0.95 \\
\hline 16 & Demostrar respeto por las normas y políticas de la Consejería. & 135 & 2 & 6 & 4.73 & 1.040 & -.520 & -.394 & $2.628 * * *$ & 0.70 & 0.95 \\
\hline \multicolumn{12}{|c|}{ 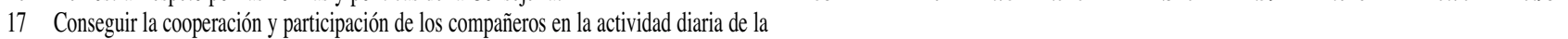 } \\
\hline & Consejería. & 135 & 1 & 6 & 4.33 & 1.125 & -.322 & -.511 & $2.490 * * *$ & 0.59 & 0.95 \\
\hline 18 & Trabajar duro con un esfuerzo superior al normal. & 135 & 1 & 6 & 4.22 & 1.176 & -.387 & -.251 & $2.038 * * *$ & 0.67 & 0.95 \\
\hline 19 & Participar responsablemente en reuniones y actividades grupales. & 135 & 1 & 6 & 4.44 & 1.163 & -.510 & .053 & $2.042 * * *$ & 0.64 & 0.95 \\
\hline 20 & Comprometerse con su propio desarrollo para mejorar su eficacia personal. & 135 & 3 & 6 & 4.96 & .909 & -.531 & -.532 & $2.728 * * *$ & 0.76 & 0.95 \\
\hline 21 & Comportarse de forma que beneficie a la Consejería. & 134 & 2 & 6 & 4.72 & 1.051 & -.333 & -.917 & $2.323 * * *$ & 0.83 & 0.95 \\
\hline 22 & Comportarse de forma que beneficie a los compañeros de la Consejería. & 135 & 2 & 6 & 4.91 & .966 & -.426 & -.643 & $2.452 * * *$ & 0.77 & 0.95 \\
\hline 23 & Ayudar a los compañeros de trabajo con sus problemas personales. & 135 & 1 & 6 & 4.45 & 1.337 & -.628 & -.482 & $2.408 * * *$ & 0.60 & 0.95 \\
\hline 24 & Prestar servicio o ayuda a los usuarios o público en general, más allá de lo normal. & 135 & 1 & 6 & 4.70 & 1.140 & -1.021 & 1.204 & $2.784 * * *$ & 0.62 & 0.95 \\
\hline 25 & Sugerir mejoras en procedimientos, administración u organización. & 135 & 1 & 6 & 4.24 & 1.218 & -.431 & -.109 & $2.055 * * *$ & 0.50 & 0.96 \\
\hline 26 & Permanecer en la Consejería a pesar de las dificultades o dureza de las condiciones. & 135 & 1 & 6 & 4.50 & 1.239 & -.780 & .360 & $2.393 * * *$ & 0.61 & 0.95 \\
\hline \multirow[t]{2}{*}{27} & Mostrar dedicación en el trabajo. & 135 & 3 & 6 & 5.14 & .839 & -.580 & -.561 & $2.871 * * *$ & 0.77 & 0.95 \\
\hline & Escala $O C B$ & 132 & 2.15 & 6.00 & 4.67 & .724 & - & .082 (.419) & .793 n.s. & & 0.96 \\
\hline
\end{tabular}

Fiabilidad: $\alpha=0.96 ; \lambda_{6}=0.97 ; \Omega_{h}=0.79 ; \Omega_{\text {hasintótica }}=0.82 ; \Omega_{t}=0.96$

No Aditividad: $\mathrm{F}(1.3563)=3.142$ (n.s.)

Estimación de Tukey de la potencia a la que es necesario elevar las observaciones para conseguir la aditividad = 1.535.

$\mathrm{T}^{2}$ de Hotelling $=449.929(\mathrm{~F}(26.106)=14.002 ; \mathrm{p} \leq 0.001)(\mathrm{H} 0$ : todos los elementos tienen la misma media $)$

$* * * \mathrm{p} \leq 0.001$

Tabla 2. Estadísticos de las soluciones unidimensional y tridimensional de la escala

\begin{tabular}{|c|c|c|c|c|}
\hline & \multirow{2}{*}{$\begin{array}{l}\text { Componente } \\
\text { Principal }\end{array}$} & \multicolumn{3}{|c|}{ Factorial } \\
\hline & & $\mathrm{F} 1$ & $\mathrm{~F} 2$ & F3 \\
\hline Valores propios & 10.30 & 1.9 & 1.3 & 1.4 \\
\hline Correlaciones Puntuaciones $\times$ Factores & 0.89 & 0.71 & 0.78 & 0.78 \\
\hline $\mathrm{R}^{2}$ múltiple de Puntuaciones $\times$ Factores & 0.79 & 0.50 & 0.60 & 0.61 \\
\hline Correlación Mínima de puntuaciones factoriales estimadas & 0.59 & 0.01 & 0.21 & 0.22 \\
\hline Ajuste (g.1.) & $7.14(324)$ & & & (273) \\
\hline$\chi^{2}(\mathrm{~N}=135)$ & $881.63^{* * *}$ & & & $71 * * *$ \\
\hline$\sqrt{\text { Residuales }}(\mathrm{g} .1)$. & $0.08(0.12)$ & & & $(0.06)$ \\
\hline RMSEA [I.C.(90\%)] & $0.121[0.104,0.122]$ & & 0.0 & $.089]$ \\
\hline BIC & -707.68 & & & 38.43 \\
\hline
\end{tabular}

$* * * \mathrm{p} \leq 0.001$

oblicuamente (rotación oblimin). Para estos análisis se usó la función de análisis factorial del toolbox “ULLRtoolbox.v.1.0.R” (Hernández-Cabrera, 2012), introduciendo la matriz de correlaciones policóricas en vez de la matriz de correlaciones de Pearson.
El determinante de $\mathbf{R}$ resultó diferente de cero, su índice de adecuación muestral es algo pobre $(\mathrm{KMO}=$ 0.682 ), aunque supera el nivel de 0.6 (Tabachnick y Fidell, 2001) y la matriz no presenta esfericidad según la prueba de Bartlett $\left(\chi^{2}(351)=3802.872 ; \mathrm{p} \leq 0.001\right)$. 
Dadas estas características se procedió con los análisis, cuyos resultados más relevantes se muestran en la Tabla 3.

Como puede verse en la Tabla 3, la solución más adecuada es la de tres factores. Sin embargo, el tercer factor sólo explica algo más del 10\% de la varianza (pesos factoriales superiores a 0.32) de los elementos 26 (Permanecer en la Consejería a pesar de las dificultades o dureza de las condiciones) y 27 (Mostrar dedicación en el trabajo), que no llegan a compartir el $10 \%$ de su varianza con los factores 1 y 2 . Además, se puede ver que el elemento 11 (No quejarse de las condiciones de trabajo en la Consejería), tiene sus pesos repartidos en los tres factores y comparte menos del $10 \%$ de la varianza con cada uno de ellos.

A la vista de estos resultados se siguió un proceso iterativo en el que se fueron eliminando elementos de los análisis que presentaban doble o triple saturación en la solución de tres factores. Este procedimiento llevó a eliminar el elemento 11 en un primer paso, además de los elementos 2 (Mostrar un esfuerzo superior al normal en su trabajo) y 6 (Seguir las normas y pro-

Tabla 3. Estructura factorial unidimensional y tridimensional de la escala completa (27 ítems)

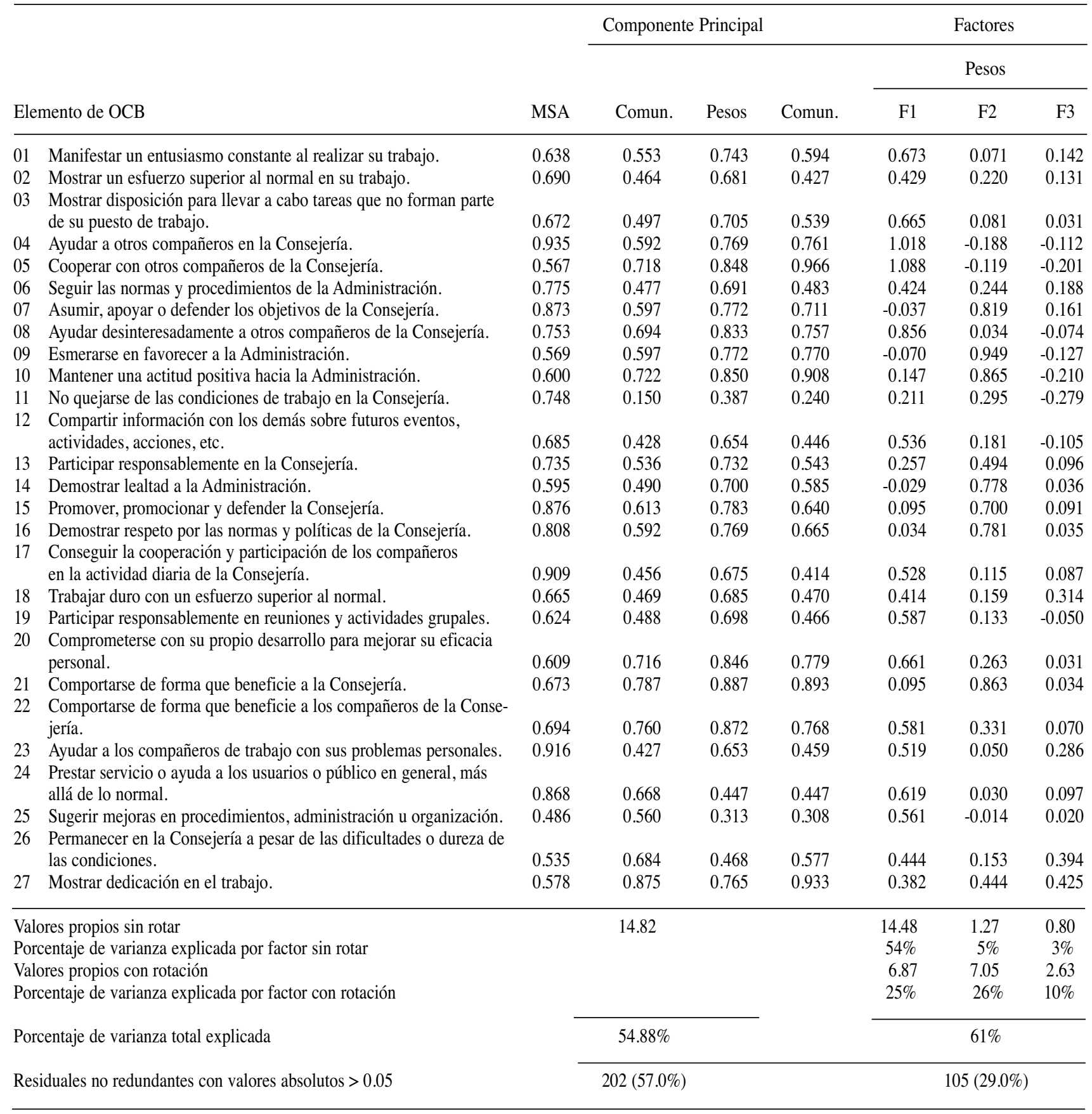


Tabla 4. Estructura factorial unidimensional y tridimensional de la escala depurada (19 ítems)

\begin{tabular}{|c|c|c|c|c|c|c|c|c|}
\hline \multirow{3}{*}{\multicolumn{2}{|c|}{ Elemento de OCB }} & \multirow[b]{3}{*}{ MSA } & \multicolumn{3}{|c|}{ Componente Principal } & \multicolumn{3}{|c|}{ Factores $(61 \%)$} \\
\hline & & & \multirow[b]{2}{*}{ Comun. } & \multirow[b]{2}{*}{ Pesos } & \multirow[b]{2}{*}{ Comun. } & \multicolumn{3}{|c|}{ Pesos } \\
\hline & & & & & & $\mathrm{F} 1$ & $\mathrm{~F} 2$ & F3 \\
\hline \multirow[t]{2}{*}{3} & Mostrar disposición para llevar a cabo tareas que no forman parte & & & & & & & \\
\hline & de su puesto de trabajo. & 0.887 & 0.493 & 0.702 & 0.507 & 0.250 & 0.457 & 0.104 \\
\hline 4 & Ayudar a otros compañeros en la Consejería. & 0.907 & 0.573 & 0.757 & 0.826 & -0.113 & 0.879 & 0.176 \\
\hline 5 & Cooperar con otros compañeros de la Consejería. & 0.783 & 0.687 & 0.829 & 0.869 & 0.106 & 0.820 & 0.070 \\
\hline 7 & Asumir, apoyar o defender los objetivos de la Consejería. & 0.898 & 0.610 & 0.781 & 0.706 & 0.876 & -0.074 & 0.017 \\
\hline 8 & Ayudar desinteresadamente a otros compañeros de la Consejería. & 0.918 & 0.678 & 0.823 & 0.802 & 0.072 & 0.710 & 0.219 \\
\hline 9 & Esmerarse en favorecer a la Administración. & 0.802 & 0.641 & 0.801 & 0.671 & 0.670 & 0.024 & 0.207 \\
\hline 10 & Mantener una actitud positiva hacia la Administración. & 0.794 & 0.753 & 0.868 & 0.845 & 0.821 & 0.222 & -0.110 \\
\hline 12 & $\begin{array}{l}\text { Compartir información con los demás sobre futuros eventos, } \\
\text { actividades, acciones, etc. }\end{array}$ & 0.931 & 0.409 & 0.640 & 0.454 & 0.331 & 0.485 & -0.131 \\
\hline 13 & Participar responsablemente en la Consejería. & 0.920 & 0.556 & 0.746 & 0.558 & 0.633 & 0.189 & -0.037 \\
\hline 14 & Demostrar lealtad a la Administración. & 0.856 & 0.526 & 0.725 & 0.573 & 0.745 & -0.014 & 0.037 \\
\hline 15 & Promover, promocionar y defender la Consejería. & 0.932 & 0.630 & 0.794 & 0.667 & 0.689 & -0.046 & 0.241 \\
\hline 16 & Demostrar respeto por las normas y políticas de la Consejería. & 0.924 & 0.618 & 0.786 & 0.699 & 0.851 & 0.035 & -0.070 \\
\hline 18 & Trabajar duro con un esfuerzo superior al normal. & 0.870 & 0.427 & 0.653 & 0.553 & 0.205 & -0.031 & 0.629 \\
\hline 20 & $\begin{array}{l}\text { Comprometerse con su propio desarrollo para mejorar su efica- } \\
\text { cia personal. }\end{array}$ & 0.803 & 0.711 & 0.843 & 0.1 & 0.450 & 0.476 & 0.032 \\
\hline 21 & Comportarse de forma que beneficie a la Consejería. & 0.826 & 0.802 & 0.896 & 0.884 & 0.855 & -0.037 & 0.177 \\
\hline 23 & Ayudar a los compañeros de trabajo con sus problemas personales. & 0.807 & 0.394 & 0.628 & 0.453 & 0.129 & 0.141 & 0.499 \\
\hline & Prestar servicio o ayuda a los usuarios o público en general, más & & & & & & & \\
\hline & allá de lo normal. & 0.911 & 0.454 & 0.673 & 0.670 & -0.096 & 0.296 & 0.687 \\
\hline \multirow[t]{2}{*}{26} & Permanecer en la Consejería a pesar de las dificultades o dureza & & & & & & & \\
\hline & de las condiciones. & 0.720 & 0.484 & 0.696 & 0.699 & 0.129 & 0.028 & 0.741 \\
\hline 27 & Mostrar dedicación en el trabajo. & 0.767 & 0.777 & 0.881 & 0.758 & 0.618 & 0.124 & 0.248 \\
\hline \multicolumn{3}{|c|}{ Valores propios sin rotar } & 11.22 & & & 10.91 & 1.14 & 0.88 \\
\hline \multicolumn{3}{|c|}{ Porcentaje de varianza explicada por factor sin rotar } & & & & $57 \%$ & $6 \%$ & $5 \%$ \\
\hline \multicolumn{3}{|c|}{ Valores propios con rotación } & & & & 6.87 & 3.98 & 2.08 \\
\hline \multicolumn{3}{|c|}{ Porcentaje de varianza explicada por factor con rotación } & & & & $36 \%$ & $21 \%$ & $11 \%$ \\
\hline \multicolumn{3}{|c|}{ Porcentaje de varianza total explicada } & $59 \%$ & & & & $68 \%$ & \\
\hline \multicolumn{3}{|c|}{ Residuales no redundantes con valores absolutos $<0.05$} & $106(61 \%)$ & & & & $23(13 \%$ & \\
\hline
\end{tabular}

1Se han invertido los pesos para facilitar la lectura e interpretación de las correlaciones

cedimientos de la Administración) en el segundo paso y los elementos 1 (Manifestar un entusiasmo constante al realizar su trabajo), 17 (Conseguir la cooperación y participación de los compañeros en la actividad diaria de la Consejería), 19 (Participar responsablemente en reuniones y actividades grupales), 22 (Comportarse de forma que beneficie a los compañeros de la Consejería) y 25 (Sugerir mejoras en procedimientos, administración u organización) en el tercer paso. Por último, se eliminó un caso por presentar una puntuación factorial extrema.

La distribución multivariada de los datos resultantes de esta depuración no puede considerarse normal, tanto para asimetría (Mardia $=135.68$; valor $=$ 2962.353; $\mathrm{p} \leq 0.001$ ) como para apuntamiento (Mardia $=506.88 ;$ valor $=21.855 ; \mathrm{p} \leq 0.001)$.

El análisis paralelo de esta matriz de correlaciones resultó en una solución muy similar a la obtenida con la matriz de correlaciones sin depurar, por lo que procedimos a extraer un componente principal (mediante un análisis $\mathrm{CP}$ ) y tres factores (mediante AF con extracción por mínimos cuadrados y rotación oblimin). El determinante de esta matriz resultó diferente de cero $(\mathrm{D}=2.82 \mathrm{E}-9)$, con una medida de adecuación muestral de Kaiser-Meyer-Olkin bastante mejorada respecto de la matriz original sin depurar $(\mathrm{KMO}=0.847)$. La prueba de esfericidad de Bartlett resultó significativa $\left(\chi^{2}\right.$ $(171)=2477.1 ; \mathrm{p} \leq 0.001)$. Además, la raíz de la contribución media al cuadrado de los factores fue inferior a $0.07(\mathrm{RMS}=0.037)$ y el ajuste fue del $98 \%$.

Como puede verse en la Tabla 4, las medidas de adecuación muestral de los elementos son aceptables, apreciándose una mejoría con respecto al análisis sin depurar (promedio de MSA $=0.856$; Máx. $=0.932$; Mín. $=0.720)$. Los porcentajes de varianza explicados también aumentaron, llegando al 59\% en la solución de un componente, y al $68 \%$ en la solución de tres factores.

Al comparar ambos análisis aplicados a esta matriz depurada, se observa que la solución del análisis 
Componentes Principales es adecuada, pero el número de residuales no redundantes sigue siendo muy elevado $(61 \%)$, aunque el porcentaje de varianza explicado pueda ser aceptable. El promedio de los pesos factoriales está en 0.726 , con un mínimo de 0.628 y un máximo de 0.896. Estos resultados apoyan la hipótesis de unidimensionalidad del constructo. Sin embargo, al aplicar el Análisis Factorial sobre esta matriz depurada, encontramos que la estructura de tres factores explica algo más de varianza común $(68 \%)$ y que el número de residuales no redundantes disminuye considerablemente hasta el $13 \%$. Esta solución de tres factores se presenta en forma resumida en la Tabla 5. tores se presentan los valores de las correlaciones (Pearson) entre éstos. Como puede verse, entre el factor 1 y 2 hay una estrecha relación $\left(\mathrm{r}_{12}=0.668\right)$, mientras que tanto el 1 como el 2 presentan correlaciones moderadas con el $3\left(r_{13}=0.478 ; r_{23}=0.481\right)$, todas ellas significativas, lo que apoyaría la existencia de una dimensión de orden superior.

Una vez salvadas las puntuaciones factoriales en estos tres factores se procedió a ejecutar un análisis factorial de segundo orden. En este caso la matriz de correlaciones utilizada se basó en Pearson, dado que las variables (puntuaciones factoriales) son continuas. El análisis paralelo de los diagramas de sedimentación

Tabla 5. Estructura factorial tridimensional de la escala depurada

\begin{tabular}{|c|c|c|c|c|}
\hline \multicolumn{2}{|c|}{ Elementos de OCB } & \multirow{2}{*}{$\begin{array}{r}\text { COM. } \\
0.876\end{array}$} & \multirow{2}{*}{$\frac{\text { FAC. }}{1}$} & \multirow{2}{*}{$\frac{\text { Coleman y Borman }}{2}$} \\
\hline 07 & Asumir, apoyar o defender los objetivos de la Consejería. & & & \\
\hline 21 & Comportarse de forma que beneficie a la Consejería. & 0.855 & & 3 \\
\hline 16 & Demostrar respeto por las normas y políticas de la Consejería. & 0.851 & & 3 \\
\hline 10 & Mantener una actitud positiva hacia la Administración. & 0.821 & & 2 \\
\hline 14 & Demostrar lealtad a la Administración. & 0.745 & & 2 \\
\hline 15 & Promover, promocionar y defender la Consejería. & 0.689 & & 2 \\
\hline 09 & Esmerarse en favorecer a la Administración. & 0.670 & & 3 \\
\hline 13 & Participar responsablemente en la Consejería. & 0.633 & & 3 \\
\hline 27 & Mostrar dedicación en el trabajo. & 0.618 & & 4 \\
\hline 04 & Ayudar a otros compañeros en la Consejería. & 0.879 & 2 & 1 \\
\hline 05 & Cooperar con otros compañeros de la Consejería. & 0.820 & & 1 \\
\hline 08 & Ayudar desinteresadamente a otros compañeros de la Consejería. & 0.710 & & 1 \\
\hline 12 & Compartir información con los demás sobre futuros eventos, actividades, acciones, etc. & 0.485 & & 1 \\
\hline 20 & Comprometerse con su propio desarrollo para mejorar su eficacia personal. & 0.476 & & 1 \\
\hline 03 & Mostrar disposición para llevar a cabo tareas que no forman parte de su puesto de trabajo. & 0.457 & & 2 \\
\hline 26 & Permanecer en la Consejería a pesar de las dificultades o dureza de las condiciones. & 0.741 & 3 & 2 \\
\hline 24 & Prestar servicio o ayuda a los usuarios o público en general, más allá de lo normal. & 0.687 & & 2 \\
\hline 18 & Trabajar duro con un esfuerzo superior al normal. & 0.629 & & 3 \\
\hline 23 & Ayudar a los compañeros de trabajo con sus problemas personales. & 0.499 & & 1 \\
\hline
\end{tabular}

Como puede verse, el primero de los factores agrupa los elementos relacionados con conductas de apoyo, respeto y lealtad a la Unidad en la que trabajan los participantes y a la Administración Pública en general. Este factor puede denominarse "Cumplimiento Generalizado" y aglutina, principalmente, elementos de los factores 2 y 3 del trabajo original de Coleman y Borman (2000), respectivamente: "Asumir, apoyar y defender los objetivos organizacionales" y "Seguir las normas y procedimientos organizacionales". El segundo factor puede llamarse "Altruismo" y agrupa los elementos relacionados con la cooperación con los compañeros. Aglutina, principalmente, elementos del primer factor de Coleman y Borman (2000) "Ayudar y cooperar con otros". Los dos primeros factores se corresponden con los encontrados por Organ (1988). El tercer factor es de difícil etiquetado, pues agrupa elementos de diversa naturaleza. En él saturan elementos de los factores primero, segundo y tercero del trabajo original. En la Figura 1 se muestra el modelo de medida de esta solución. En las conexiones entre fac- recomienda una solución de un único componente y factor, por lo que se eligió aplicar un Análisis Factorial con extracción de un único factor. Los valores de Mardia para la normalidad multivariada resultaron significativos, tanto para la asimetría (Mardia $=2.382$; valor $=51.998 ; \mathrm{p} \leq 0,001)$, como para el apuntamiento $($ Mardia $=19.332$; valor $=4.526 ; \mathrm{p} \leq 0.001)$, aunque no tan elevados como en los casos anteriores. La prueba de esfericidad de Bartlett nos indica que la matriz no es esférica $\left(\chi^{2}(3)=9.505 ; \mathrm{p} \leq 0.001\right)$; la medida de adecuación muestral de Kaiser-Meyer-Olkin resultó adecuada $(\mathrm{KMO}=0.653)$; la raíz de la contribución media al cuadrado de los factores fue inferior a 0.07 $(\mathrm{RMS}=0.001)$ y el ajuste fue del $82 \%$. Las medidas de adecuación muestral de cada variable (factor primario) fueron, respectivamente, MSA (Cumplimiento) = $0.620 ;$ MSA (Altruismo) $=0.623 ;$ ySA $_{\mathrm{F} 3}=0.777$. En la Figura 2 se muestra el modelo numérico de la solución obtenida.

El factor de segundo orden resultante tuvo un valor propio de 1.52 y explicó un $51 \%$ de la varianza de $\mathbf{R}$. 
Figura 1. Modelo de medida de la solución factorial tridimensional

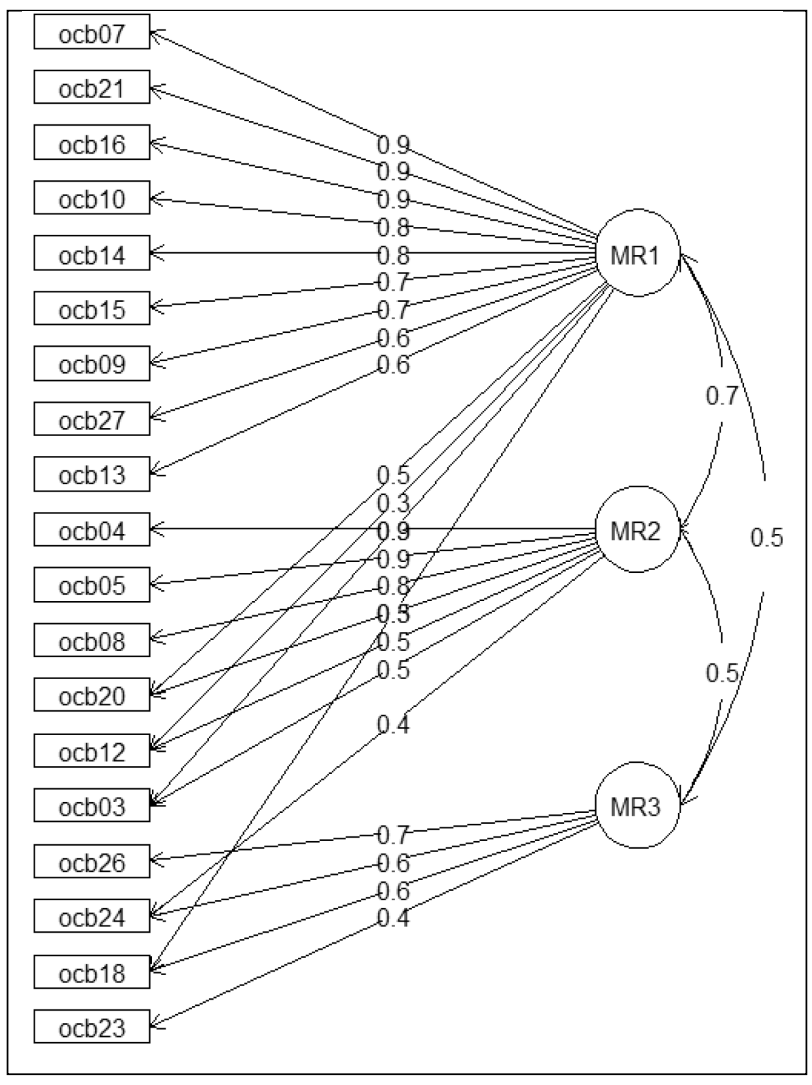

Figura 2. Modelo de medida de la solución unidimensional

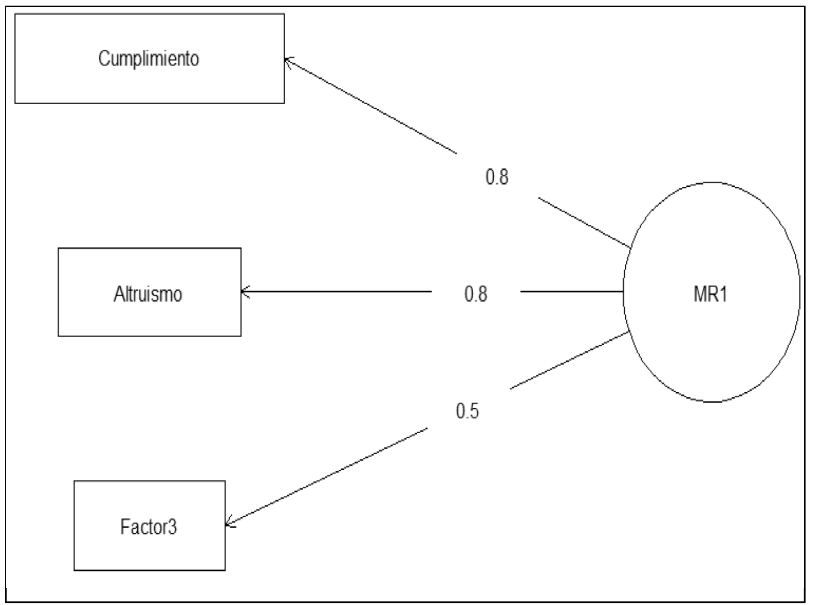

Atendiendo a las comunalidades, los factores primarios 1 y 2 alcanzan valores de 0.796 y 0.777 , respectivamente, mientras que el tercero sólo llega a 0.532 $(0.8,0.8$ y 0.5 en la Figura 2). Esta situación se refleja también en los pesos factoriales, de manera que los dos factores primarios Cumplimiento y Altruismo tienen pesos de 0.467 y 0.422 respectivamente, mientras que $\mathrm{F}_{3}$ tiene un peso de 0.160 . Estos resultados apoyan la hipótesis del constructo latente medido de forma imperfecta por varias dimensiones.

\section{Discusión y Conclusiones}

Los resultados de este trabajo indican que el cuestionario de desempeño cívico adaptado al español y aplicado a una muestra de empleados públicos tiene una alta consistencia interna. Sin embargo, su análisis en profundidad nos indica la existencia de tres factores subyacentes. Dados estos resultados se aplicaron análisis factoriales y se encontró una estructura de tres factores de primer orden y un factor latente de segundo orden.

Los resultados obtenidos muestran una estructura de dos dimensiones o factores claramente congruentes con los resultados obtenidos en investigaciones previas (Coleman y Borman, 2000; Hogan et al., 1998; Werner, 1994) y que aquí se han denominado como Cumplimiento Generalizado y Altruismo, siguiendo la terminología utilizada por Organ (1988). El tercer factor es de difícil interpretación y no se ha etiquetado. Los elementos que saturan en este tercer factor no parecen apresar facetas de desempeño relacionadas teóricamente. La aparición de este factor puede deberse a la distribución no normal de los datos (falta de normalidad) o bien al limitado número de casos estudiados. A pesar de esto, los dos primeros factores obtenidos tienen una alta consistencia teórica, lo que indica que es muy probable que la escala mida dos factores reales y el tercero sea un residuo artefactual.

Coleman y Borman (2000) presentan una solución de tres factores. Sin embargo, estos autores llegan a esta estructura siguiendo un método racional. Este método se aplicó a los resultados de tres técnicas estadísticas diferentes aplicadas a juicios de expertos (no evaluaciones empíricas). Es por ello que consideramos que los datos aportados en la presente investigación dan una imagen más realista de la estructura del cuestionario y también del constructo, en la medida en que éste es representativo del desempeño cívico. Este resultado está también en línea con las aportaciones de Organ (1988), Werner (1994) o Hogan et al. (1998), el desempeño cívico parece tener dos componentes claros, uno relacionado con el apoyo social y otro con el cumplimiento y lealtad a la organización.

Además, los resultados indican que las dimensiones obtenidas están moderadamente relacionadas. Un análisis factorial de segundo orden sobre las correlaciones entre estos tres factores de primer orden resultó en una única dimensión. Este resultado apoya la hipótesis del constructo latente. Con estos resultados se puede apoyar la idea de la existencia de un único constructo de desempeño cívico que englobaría y explicaría las dos dimensiones anteriormente descritas (Hoffman et al., 2007; LePine et al., 2002).

De cara a la generalización de los resultados, es importante destacar que las distribuciones de las respuestas a los elementos se muestran sesgadas hacia el extremo de mejor desempeño. Este problema no es nuevo, aunque no hay muchos trabajos en la literatura que lo hagan patente. Una de las causas más probables 
de los sesgos encontrados en este trabajo es la deseabilidad social, concretamente la dimensión de distorsión de la imagen. Salgado (2005) examinó los problemas asociados a la distorsión de respuestas y deseabilidad social en situaciones de evaluación de personalidad en contextos organizacionales. Según este autor, la deseabilidad social afecta a las medidas de evaluación de la personalidad basadas en cuestionarios. De la misma manera, la deseabilidad social puede estar influyendo las respuestas a las medidas de desempeño basadas en autoinformes.

En esta línea, Kroger y Wood (1993) pusieron de manifiesto el hecho de que la posibilidad de falsear las respuestas en pruebas de personalidad genera la cuestión de si estos tests están midiendo disposiciones permanentes o representaciones momentáneas del self. Existe abundante investigación sobre este tema (Christiansen, Goffin, Johnston y Rothstein, 1994; Dunnette, McCartney, Carlson y Kirchner, 1962; Ellingson, Smith y Sackett, 2001; Hough, Eaton, Dunnette, Kamp y McCloy, 1990; Mueller-Hanson, Heggestad y Thornton III, 2003; Seibel, Wallbrown, Reuter y Barnett, 1990). Sin embargo, no ocurre lo mismo en el ámbito de la evaluación del desempeño. Futuros trabajos deberían interesarse en esta posible causa de distorsión de respuestas y, lo que es más relevante para el desarrollo conceptual, de la inflación de correlaciones entre ítems y dimensiones.

Además, debe considerarse la varianza debida al método como un aspecto de este trabajo que podría mejorarse. Siguiendo a LePine et al. (2002), el uso del mismo cuestionario e informante produce varianza debida al método (correlated method variance). Esto es, el factor de segundo orden que hemos encontrado podría deberse al uso de un único cuestionario aplicado a un solo tipo de evaluadores. En siguientes trabajos se debería intentar explorar la estructura de primer y segundo orden sobre datos obtenidos, tanto con auto evaluaciones, como a partir de evaluaciones realizadas por supervisores y/o pares.

Este trabajo concluye con una escala válida (en la medida en que su estructura es adecuada a la teoría), relativamente corta y fiable, que puede ser de utilidad en la práctica profesional. Aunque la solución de tres factores sea la más adecuada, la solución de un solo factor no es desdeñable. Los resultados indican que la escala puede ser utilizada para medir desempeño cívico como constructo global, obteniendo una única puntuación media para cada trabajador, pero también es adecuada su utilización para medir diferentes aspectos de este constructo. Esto permitiría al profesional elegir una u otra solución dependiendo de los objetivos de la intervención. Por ejemplo, un factor para evaluación del desempeño y tres factores para desarrollar objetivos de formación. Además, los resultados presentados abren nuevas vías de investigación, que podrían centrarse tanto en la estructura como en las variables que pueden estar afectando a las respuestas.

\section{Extended Summary}

Research on work performance highlights the importance of including all kind of behaviors relevant to organizational outcomes (Salgado \& Cabal, 2011). These behaviors are grouped in three dimensions: task performance, citizenship performance, and counterproductive behaviors (Ployhart, Schneider, \& Schmitt, 2006; Salgado \& Cabal, 2011; Viswesvaran \& Ones, 2000).

This article focuses on citizenship performance, studying its structure through the adaptation into Spanish of the questionnaire used by Coleman and Borman (2000). Citizenship performance is defined as the enactment of a set of discretionary behaviors, generally not part of the formal role, and not explicitly recognized in compensation systems (Bateman \& Organ, 1983; Borman \& Motowidlo, 1997; Organ, 1988; Organ \& Ryan, 1995; Ployhart et al, 2006). They contribute to the maintenance of the social system, facilitating the achievement of organizational goals (Borman \& Motowidlo, 1993; Witman, Van Rooy, \& Viswesvaran, 2010).

There are two hypotheses on citizenship performance structure. Some authors assume several dimensions within the construct. Coleman and Borman (2000) proposed three major dimensions of citizenship performance: interpersonal, organizational, and job/task related. Werner (1994) previously found two dimensions of citizenship performance (OCBO, oriented to benefit the organization; and OCBI oriented to benefit individuals in the organization), corresponding to Generalized Compliance and Altruism found by Smith, Organ, and Near (1983). In the same line, Hogan, Rybicki, Motowidlo, and Borman (1998), found two factors: Dedication to work, and Interpersonal Facilitation. Other authors, like Hoff-man, Blair, Meriac, and Woehr (2007) assumed the hypothesis of unidimensionality. In this line, Poropat and Jones (2009) found a single factor solution.

The examples revised show some problems in the field: lack of a rigorous terminology, discrepancies on the structure of the construct, and absence of a set of items suitable to be incorporated in a citizenship performance questionnaire.

A meta-analysis by LePine et al. (2002) concluded that citizenship performance must be conceptualized as a latent construct redefined as ".... a general tendency to be cooperative and helpful in organizational settings" (p.61). The assumption that there is a latent construct implies high correlations between OCB dimensions, and a second order factor analysis should result in a unique factor. Hoffman et al. (2007) broadened the meta-analytic revision of OCB. Their confirmatory 
factor analysis supported a single factor structure. From these results, they suggest that sub dimensions of OCB are indicators of a more general construct.

When studying the structure of citizenship performance, reliability and factor analyses are necessary. The majority of studies report Cronbach's alpha, obviating the fact that $\alpha$ can be an inappropriate indicator of data structure. Revelle and Zinbarg (2009) suggested to use McDonald's Omega total - $\omega_{t}(1978)$ and Omega hierarchical $-\omega_{\mathrm{h}}(1999)$. Omega total refers the proportion of variance due to all factors common to the items. When $\omega_{t}=\alpha$, the questionnaire can be considered unidimensional, supporting the aggregation hypothesis. Omega hierarchical denotes the proportion of test variance due to a unique general factor. It refers to the accuracy with which test total scores estimate a common latent variable. A high $\omega_{\mathrm{h}}$ would support the aggregation hypothesis.

The aggregation hypothesis would have more support when single factor solutions had better fit than multiple factors solutions. On the other hand, if multiple factor solutions generate correlated factors, the hypothesis of the latent factor would be supported. Unfortunately, excepting the cases where structural equations are applied, goodness of fit is seldom reported. Also, when factor analyses are reported, what in fact have been applied are principal components analyses, mostly with orthogonal rotations.

In this work we attempt to offer a Spanish version of a questionnaire as representative as possible of Coleman and Borman's (2000) one. We also try to deepen in the structure of citizenship performance, using adequate statistical tools applied to real-life data.

\section{Method}

\section{Participants}

The sample comprised the whole staff (135 employees) from a Unit in the Public Administration, dedicated to facilitate the touristic development of the Canary Islands. This Unit was structured in 18 subunits and 25 different jobs. These jobs were grouped in 8 families: Administrative staff (37\%); Riggers (2.2\%); Inspectors $(3.7 \%)$; Section/Service chiefs $(22.2 \%)$; Computer operators $(3.7 \%)$; Secretaries $(4.4 \%)$; Subordinates $(8.9 \%)$; and Senior Technicians (17.8\%).

\section{Instrument}

Item adaptation into Spanish was carried out in several phases. Researchers translated the items and a committee of Public Administration experts assessed the clarity of language and proposed alternatives. The final version included 27 items with 6 points graphic scales and two extreme anchors. Participants were asked to answer each question depending on what they believed that better described their behavior at work.

\section{Procedure}

Each employee received a questionnaire and gave it back to the research team confidentially. Response tendency was analyzed following several criteria and those who did not pass two or more criteria were informed and visited by a member of the research team, so they could change or keep their responses.

\section{Data analysis}

This study had two goals that were dealt with two different methodologies. To analyze reliability we applied Omega hierarchical $\left(\omega_{h}\right)$ and Omega total $\left(\omega_{t}\right)$ and Guttman's $\lambda_{6}$ tests were chosen. To analyze the structure of citizenship performance we carried out a procedure of factor analyses on the policoric correlation matrix, and a second order factor analysis on the first order factor scores. Policoric correlations are adequate when ordinal responses of participants are assumed to be a reflection of the elections they make from a continuous conception of the measured construct (Choi, Peters, \& Mueller, 2010; Ekström, 2008, 2009; Juras \& Pasaric, 2006; Uebersax, 2006). This is the case when we use Likert type scales with integer values.

\section{Results}

Table 1 shows the distributional statistics and reliability results. Hotelling's $\mathrm{T}^{2}$ resulted significant, so means across items are not equal. None of the distributions can be considered normal, as all tests of normality resulted significant.

Reliability is high when we consider Cronbach's coefficient $(\alpha=0.96)$, and additivity is adequate. All the alternative reliability indexes indicate there is a single factor (Guttman's $\lambda_{6}$ was slightly higher than $\alpha ; \omega_{\mathrm{h}}$ value indicates that a unique factor explains $78 \%$ of items variance; $\omega_{\mathrm{t}}$ equals $\alpha$ ). Nevertheless, the same reliability analysis procedure carried out with the software package R (R Development Core Team, 2008), recommended a three factors alternative solution with better fitting indexes (Table 2).

Parallel analysis applied to $\boldsymbol{R}_{\text {(policoric) }}$ suggested the extraction of one principal component and three factors. A PC analysis extracting a unique component, and a FA extracting three factors (minimum squares), with oblique rotation were performed. For these analyses we used the FA function from the toolbox "ULLRtoolbox.v.1.0.R" (Hernández-Cabrera, 2012).

Matrix properties were adequate for factorization (Det. $>0 ; \mathrm{KMO}=0.682 ;$ Bartlett's $\chi^{2}(351)=$ 
3802.872; $\mathrm{p} \leq 0.001)$. Table 3 shows the three factors solution is more adequate for the raw data matrix. Nevertheless, an iterative procedure was carried out through which items showing double or triple loadings were eliminated from the analyses. Throughout this procedure, 8 items and one extreme case were removed.

Parallel analysis applied to the cleaned matrix resulted in a similar solution to the previous one, so a $\mathrm{PC}$ analysis and a FA were performed. Matrix properties were adequate (Det. $>0 ; \mathrm{KMO}=0.847$; Bartlett's $\left.\chi^{2}(171)=2477.1 ; \mathrm{p} \leq 0.001\right)$. Besides, RMS $=0.037$ and fit was of $98 \%$.

Table 4 shows items' measures of sampling adequacy, which are acceptable and show an improvement with respect to the unscreened matrix (Average = 0.856; $\operatorname{Max}=0.932 ;$ Min $=0.720$ ). Explained variance also improved: $59 \%$ for PC and $68 \%$ for FA.

In the screened matrix PC, the number of non-redundant residuals is still very high (61\%). FA results (Table 5), show that the three factors structure explains some more variance and the number of non-redundant residuals decrease dramatically (13\%). First factor, Generalized Compliance, coincides with Coleman and Borman's (2000) second and third categories. Second factor, Altruism, groups items from Coleman and Borman's (2000) first category. These two factors resemble those found by Organ (1998), while the third one has a difficult interpretation, loading items from all the original categories. Figure 1 shows the measurement model of this solution. Correlation between factors 1 and 2 is moderate $\left(r_{12}=0.668\right)$, while correlations between factors 3 and 1 , and between factors 3 and 2, are lower $\left(r_{13}=0.478 ; r_{23}=0.481\right)$, all of them significant.

A second order FA was performed on first order factor scores. Parallel analysis recommended one factor, so a FA was applied. Matrix properties were adequate $\left(\right.$ Bartlett's $\chi^{2}(3)=9.505 ; \mathrm{p} \leq 0.001 ; \mathrm{KMO}=0.653$; RMS $=0.001 ;$ and fit of $82 \%)$. Measures of sampling adequacy of each variable (primary factor) were, MSA $($ Compliance $)=0.620 ;$ MSA $($ Altruism $)=0.623$; and MSA $(F 3)=0.777$. Figure 2 shows the numeric model of this solution.

The resulting factor had an eigenvalue of 1.52 and explained a $51 \%$ of variance. Primary factors General Compliance and Altruism reached communalities of 0.796 and 0.777 respectively, while factor 3 barely surpassed 0.5 (Figure 2). Compliance and Altruism load 0.467 and 0.422 respectively, while $F_{3}$ has a loading of 0.160 . These results support the hypothesis of the latent construct imperfectly measured by various dimensions.

\section{Discussion and Conclusions}

Results show that the Spanish adaptation of the Citizenship Performance questionnaire has a high internal consistency. Nevertheless, a deeper analysis shows the existence of three underlying factors. Factor analyses were performed resulting in a three factors structure, and a single second order factor solution.

Two of the primary factors are clearly consistent with previous research (Coleman \& Borman, 2000; Hogan et al., 1998; Werner, 1994), and following Organ (1988), were named Generalized Compliance and Altruism. Third factor is difficult to interpret, and was not labeled. The emergence of this factor may be due to a bad distribution of data, or to a reduced number of cases. Despite these problems, first two factors have high theoretical consistency, what suggest there are two factors and the third one is just a residual.

Following a rational methodology, Coleman and Borman (2000) suggested a structure of three categories. We consider the current results give a more realistic view of the questionnaire structure and of the construct itself: two components, one related to social support and another to organizational compliance and loyalty. These results also show a single second order factor, supporting the idea of a unique Citizenship Performance construct (Hoffman et al., 2007; LePine et al., 2002), including and explaining both first order dimensions.

It is important to emphasize that responses were biased toward the high-performance end of the scale. Although not many studies in the field make this clear, social desirability can be influencing self-reported performance ratings. Salgado (2005) examined this topic in personality assessment settings, and he suggested that social desirability affects personality measurement characteristics (e.g., reliability, construct validity). Despite the amount of research on this topic (Christiansen, Goffin, Johnston, \& Rothstein, 1994; Dunnette, McCartney, Carlson, \& Kirchner, 1962; Ellingson, Smith, \& Sackett, 2001; Hough, Eaton, Dunnette, Kamp, \& McCloy, 1990; Mueller-Hanson, Heggestad, \& Thornton III, 2003; Seibel, Wallbrown, Reuter, \& Barnett, 1990), it is not well examined in the case of performance appraisal. Future research should put more effort in debugging the role of social desirability on the possible inflation of correlations between items and between dimensions.

It should also consider correlated method variance as a point to improve in this work. Our second order factor could be the effect of using a single questionnaire applied to a single kind of assessors. Future research should try to explore the first and second order structures of data obtained from self-evaluations as well as from supervisors and peers.

This work concludes with a valid scale (as long as its structure is adequate to the theory), relatively short and reliable, that can be useful for practitioners. Although the three factors solution seems to be more adequate, the single factor one is not negligible. Therefore, the questionnaire can be utilized to measure citizenship performance as a global construct, but also it is ade- 
quate for measuring those two aspects of the construct. This would allow the professional to choose one or the other solution depending on the intervention goals: using a single factor for performance appraisal and three factors for developing training goals. Moreover, our results open new ways of research interested in the structure as well as in variables affecting responses.

\section{Referencias}

Bateman, T. S. y Organ, D. W. (1983). Job Satisfaction and the Good Soldier: The Relationship Between Affect and Employee "Citizenship". Academy of Management Journal, 26, 587-595.

Borman, W. C. y Motowidlo, S. (1993). Expanding the criterion domain to include elements of contextual performance. En N. Schmitt, \& W. C. Borman (Eds.), Personnel selection in organizations (pp. 71-98). San Francisco, CA: Jossey - Bass.

Borman, W. C. y Motowidlo, S. J. (1997). Task performance and contextual performance: The meaning for personnel selection research. Human Performance, 10, 99-109.

Borman, W. C., Penner, L. A., Allen, T. D. y Motowidlo, S. J. (2001). Personality predictors of citizenship performance. International Journal of Selection and Assessment, 9, 52-69.

Choi, J., Peters, M. y Mueller, R. O. (2010). Correlational analysis of ordinal data: from Pearson's $r$ to Bayesian polychoric correlation. Asia Pacific Education Review, 11, 459466.

Christiansen, N. D., Goffin, R. D., Johnston, N. G. y Rothstein, M. G. (1994). Correcting the 16PF for faking: Effects on criterion-related validity and individual hiring decisions. Personnel Psychology, 47, 847-860.

Coleman, V. I. y Borman, W. C. (2000). Investigating the underlying structure of the Citizenship Performance domain. Human Resource Management Review, 10, 2544.

Dorsey, D. W., Cortina, J. M. y Luchman, J. (2010). Adaptive and citizenship-related behaviors at work. En J. L. Farr, \& N. T. Tippins (Eds.), Handbook of employee selection (pp. 463-487). New York: Routledge.

Dunnette, M. D., McCartney, J., Carlson, H. C. y Kirchner, W. K. (1962). A study of faking behavior on a forced choice self-description checklist. Personnel Psychology, $15,13-24$

Ekström, J. (2008). A Generalized Definition of the Polychoric Correlation Coefficient. Obtenido de UCLA Statistics electronic publications: http://preprints.stat. ucla.edu/

Ekström, J. (2009). An empirical polychoric correlation coefficient. Obtenido de UCLA Statistics electronic publications: http://preprints.stat.ucla.edu/

Ellingson, J. E., Smith, D. B. y Sackett, P. R. (2001). Investigating the influence of social desirability on Personality factor structure. Journal of Applied Psychology, 86, 122-133.
Hernández-Cabrera, J. A. (2012). ULL R Toolbox. Obtenido de https://sites.google.com/site/ullrtoolbox

Hoffman, B. J., Blair, C. A., Meriac, J. P. y Woehr, D. J. (2007). Expanding the criterion domain? A Quantitative review of the OCB literature. Journal of Applied Psychology, 92, 555-566.

Hogan, J., Rybicki, S. L., Motowidlo, S. J. y Borman, W. C. (1998). Relations between contextual performance, personality, and occupational advancement. Human Performance, 11, 189-207.

Hough, L. M., Eaton, N. K., Dunnette, M. D., Kamp, J. D. y McCloy, R. A. (1990). Criterion-related validities of personality constructs and the effect of response distortion on those validities. Journal of Applied Psychology, 75, 581-595.

Juras, J. y Pasaric, Z. (2006). Application of tetrachoric and polychoric correlation coefficients to forecast verification. Geofisika, 23, 59-82.

Kroger, R. O. y Wood, L. A (1993). Reificatiom Faking, and the Big Five. American Psychologist, 48, 1297-1298.

LePine, J. A., Erez, A. y Johnson, D. E. (2002). The Nature and Dimensionality of Organizational Citizenship Behavior: A Critical Review and Meta-Analysis. Journal of Applied Psychology, 87, 52-65.

McDonald, R. P. (1978). Generalizability in factorable domains: "domain validity and generalizability". Educational and Psychological Measurement, 38, 75-79.

McDonald, R. P. (1999). Test theory: A unified treatment. Hillsdale, NJ: Erlbaum.

Motowidlo, S. J. y Schmit, M. J. (1999). Performance assessment in unique jobs. En D. R. Ilgen, \& E. D. Pulakos (Eds.) The changing nature of performance: Implications for staffing, motivation, and development (pp. 56-87). San Francisco, CA: Jossey-Bass.

Mueller-Hanson, R., Heggestad, E. D. y Thornton III, G. C. (2003). Faking and selection: Considering the use of personality from select-in and select-out perspectives. Journal of Applied Psychology, 88, 348-355.

Organ, D. W. (1988). Organizational citizenship behavior: The good soldier syndrome. Lexington, MA: Lexington Books.

Organ, D. W. y Ryan, K. (1995). A meta-analytic review of attitudinal and dispositional predictors of organizational citizenship behavior. Personnel Psychology, 48, 775802.

Ployhart, R. E., Schneider, B. y Schmitt, N. (2006). Staffing organizations. Contemporary practice and theory $\left(3^{\circ}\right.$ ed.). Mahwah, NJ: Lawrence Erlbaum Associates.

Poropat, A. E. y Jones, L. (2009). Development and validation of a unifactorial measure of citizenship performance. Journal of Occupational and Organizational Psychology, $82,851-869$.

R Development Core Team. (2008). A language and environment for statistical computing. Vienna, Austria: $\mathrm{R}$ Foundation for Statistical Computing.

Revelle, W. y Zinbarg, R. E. (March 2009). Coefficients Alpha, Beta, Omega, and the glb: Comments on Sijtsma. Psychometrika, 74, 145-154. 
Salgado, J. F. (2005). Personalidad y deseabilidad social en contextos organizacionales: implicaciones para la práctica de la Psicología del Trabajo y las Organizaciones. Papeles del Psicólogo, 26, 115-128.

Salgado, J. F. y Cabal, A. L. (2011). Evaluación del desempeño en la Administración Pública del Principado de Asturias: Análisis de las propiedades psicométricas. Revista de Psicología del Trabajo y de las Organizaciones, 27, 75-91.

Seibel, H. P., Wallbrown, F. H., Reuter, E. K. y Barnett, R. W. (1990). Further evidence concerning motivational distortion on the sixteen personality factor primaries by male felons. Journal of Personality Assessment, 55, 367375.

Smith, C. A., Organ, D. W. y Near, J. P. (1983). Organizational citizenship behavior: Its nature and antecedents. Journal of Applied Psychology, 68, 653-663.
Tabachnick, B. G. y Fidell, L. S. (2001). Using Multivariate Statistics (4th Edition). Needham Heights, MA: Allyn and Bacon.

Uebersax, J. S. (2006). Introduction to the Tetrachoric and Polychoric Correlation Coefficients. Obtenido de http:// www.john-uebersax.com/ stat/tetra.htm

Viswesvaran, C. y Ones, D. S. (2000). Perspectives on models of job performance. International Journal of Selection and Assessment, 8, 216-226.

Werner, J. M. (1994). Dimensions That Make a Difference: Examining the Impact of In-Role and Extra-role Behaviors on Supervisory Ratings. Journal of Applied Psychology, 79, 98-107.

Witman, D. S., Van Rooy, D. L. y Viswesvaran, C. (2010). Satisfaction, citizenship behaviors, and performance in work units: A meta-analysis of collective construct relations. Personnel Psychology, 63, 41-81.

Manuscrito Recibido: 12/09/2012

Revisión Recibida: 22/11/2012

Aceptado: 26/11/2012 